\title{
An Efficient Key-Update Scheme for Wireless Sensor Networks
}

\author{
Chien-Lung Wang ${ }^{1}$, Gwoboa Horng ${ }^{1}$, Yu-Sheng Chen ${ }^{1}$, and Tzung-Pei Hong ${ }^{2}$ \\ ${ }^{1}$ Department of Computer Science, National Chung-Hsing University \\ Taichung 40227, Taiwan, R.O.C. \\ \{phd9004, gbhorng, s9356047\}@cs.nchu.edu.tw \\ ${ }^{2}$ Department of Electrical Engineering, National University of Kaohsiung \\ Kaohsiung 811, Taiwan, R.O.C. \\ tphong@nuk. edu.tw
}

\begin{abstract}
A novel key-update scheme is proposed for wireless sensor networks. The center server in a wireless sensor network first broadcasts a series of randomly generated code slices to sensor nodes. Upon receiving all the code slices, the sensor nodes find their neighboring coordinators to generate a permutation of slice numbers and send this permutation back to the center server. The center server and the sensor nodes can thus assemble a common program based on the permutation to derive their common key. Subsequent key-updates can then be done by this program based on the previous keys. The proposed scheme is simple, efficient, and is secure if the sensor nodes cannot be compromised within a short bound of time.
\end{abstract}

\section{Introduction}

Sensor networks are a kind of ad-hoc networks [3] and widely used in real applications. In a sensor network, each sensor node is deployed in a different location and is in charge of perceiving local information and reporting to the center server. A sensor node is usually limited by its computing power, memory, and battery power. These constraints make public-key algorithms infeasible for sensor nodes. In the past two decades, a lot of researches about security protocols [1] were proposed, including several key pre-distribution schemes [2][4][5].

In real applications, sensor nodes are usually deployed in a large number in order to cover a sufficiently large area. For instance, a military aircraft may scatter a lot of tiny sensor nodes over a certain terrain to gather information. Tens of thousands of sensor nodes may be required in this case. Since the amount of sensor nodes used in an application is usually large, it is better for them to be provided as cheaply as possible. If all sensor nodes used in an application are the same, they can be manufactured uniformly and the production costs can thus be reduced. Specifically, "uniform" means that each sensor node is equipped with the same hardware, software, and initial settings. It may be criticized that each sensor node with the same keys are dangerous. This thus causes a trade-off issue between security and production cost.

An efficient key-update scheme is necessary if all sensor nodes are initially equipped with the same keys. The keys should be updated immediately after the 
deployment of sensor nodes since a compromise of the initial key of a sensor node may crash the entire sensor network. Besides, a good key-update scheme should be prompt and efficient. In this paper, a key-update scheme is thus proposed for wireless sensor networks.

\section{The Proposed Key-Update Scheme}

The proposed key-update scheme is divided into two parts: server part and sensor part. In this paper, all sensor nodes are assumed the same except for their IDs. The center server and all sensor nodes initially share a key $K_{c}$. This key is only used to initialize encryption keys and should be annihilated as soon as the deployment is done. To prevent the catastrophic consequence of compromising a sensor node, a short time bound $t$ is set for the key-update phase. Each sensor node will begin a timer immediately after its physical deployment. If a sensor node does not finish its keyupdate phase within time $t$, it should sacrifice himself. That is, it will annihilate its initial key and stop its functionalities for keeping the security of the whole sensor network. The initial key can thus be protected in this way. The proposed scheme needs to estimate the time required to compromise a sensor node and chooses $t$ as small as possible. The proposed key-update scheme executed respectively in the center server and sensor nodes is described below.

\section{Execution on the Center Server - Broadcasting Code Slices}

The center server first prepares some operators to generate random code slices. These operators are pairwise non-commutative. A code slice is composed of an operand and an operator. An operand can be any integer and an operator can be one of the following operators: addition, multiplication, division, exponentiation, logarithm, shift, etc. For example, $+2, * 4, \wedge 3$ are possible code slices and six possible combinations can be derived from the three code slices without repetition. Six possible programs can thus be assembled in the example. In general, if there are $m$ code slices, then $m$ ! possible programs can be obtained. Finally, the center server broadcasts these code slices to the wireless sensor network.

\section{Execution on the Sensor Nodes - Coordinating and Assembling}

In a sensor network, a sensor node can only communicate directly with its neighbors within a short range. A header is usually chosen from a subnet of sensor nodes as a relay. In the proposed scheme, the one with the most neighbors is elected as the header. In the dark, frogs cry to locate and identify other frogs. Similarly, each sensor node can send out its own ID to notify its neighbors. At the first stage, each sensor node sends out its ID and counts the number of its neighbors. At the second stage, each sensor node announces this number. Based on this information, headers can be elected and located by their neighbors (see Fig. 1). This method is called an echo algorithm. 


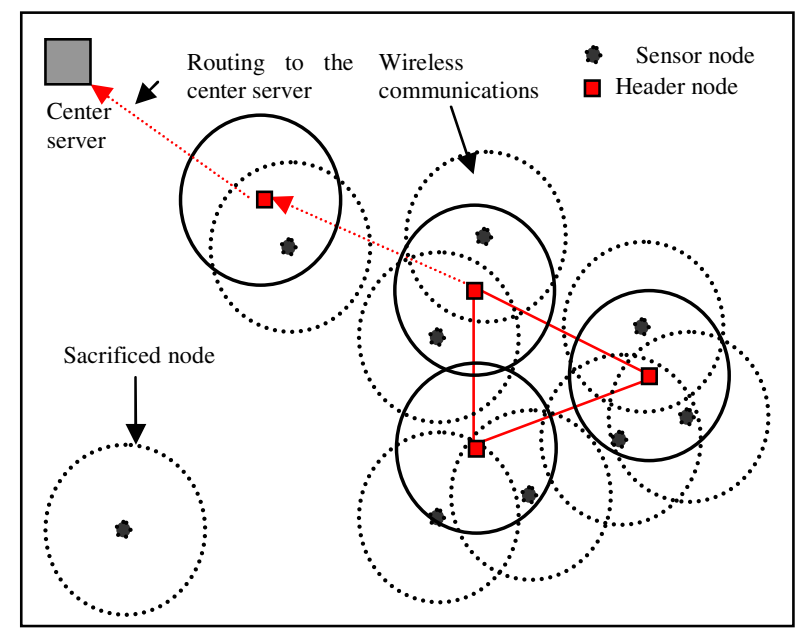

Fig. 1. System architecture: a center server and randomly distributed sensor nodes in a wireless sensor network. A solid circle represents the subnet of a certain header elected by its neighbor nodes. When a node needs to send a message to the center server, the message is first sent to the header of its subnet, then the header routes the message to the center server.

After headers are elected from sensor nodes, the next step is to form a secret program. Let the subnet of a header be defined as the network formed from the header itself and all its neighbors. Thus, there are many subnets in a wireless sensor network. Some subnets may overlap.

Each header will generate a random permutation of $m$ objects, denoted by a string $r_{m}$, upon receiving code slices $P_{l}$ to $P_{m}$ from the center server. It then deliveries $r_{m}$ to its subnet and routes a path to the center server. With the permutation $r_{m}$, the code slices can then be assembled together to get a common program $P . r_{m}$ should also be encrypted with the key $K_{c}$ before it is sent out to prevent eavesdropping.

After an agreement of the program $P$ has been made between the center server and the subnet of a certain header, subsequent key-updates can be done by executing the program $P$ on the previous key of the subnet, i.e. $k_{j}=P\left(k_{j-1}\right)$. Initially, the key of all subnets is $k_{c}=k_{0}$. But after the key-update procedure, each subnet should have its own key agreed with the center server.

\section{Security Analysis}

In the proposed scheme, an attacker may tape all the code slices $P_{l}, \ldots, P_{m}$ and try to recover a secret program $P$ of a certain subnet by rearranging the slices. But lacking the permutation information $r_{m}$, the attacker can only guess possible ones. Since each permutation of the code slices can result in an executable program, the probability for a guessed program to equal the program $P$ is only $1 / m !$. This is why the $m$ code slices are required to be pairwise non-commutative; otherwise, the probability may be larger than $1 / m$ !. The security of the proposed protocol thus depends on the size of $m . m$ may 
be increased for better security at the cost of program-assembling time. For instance, $m !>2^{64}$ for a choice of $m=21$, and $m !>2^{128}$ for a choice of $m=35$.

Suppose that the average number of neighbor nodes is $k$. Each node in a subnet then needs $2 *(1+k)$ operations. Each header node needs an extra encryption. Each node can thus easily assemble the program $P$ from the $m$ code slices. The choice for the size of $m$ provides the flexibility between security and efficiency.

\section{Conclusion}

A novel key-update scheme has been proposed for wireless sensor networks. The proposed scheme is simple, efficient and secure if the sensor nodes are assumed not to be compromised within a short time bound $t$. It is thus feasible for the proposed scheme to be applied to a resource-constrained wireless sensor network. In the future, we will attempt to improve the proposed scheme with other constraint considerations.

\section{Acknowledgement}

This research was supported by the National Science Council of the Republic of China under contract NSC- 94- 2213- E- 005- 028.

\section{References}

1. Perrig, A., Szewczyk, R., Wen, V., Culler, D., Tygar, J.D.: SPINS: Security Protocols for Sensor Networks. The Seventh ACM Annual International Conference on Mobile Computing and Networking (2001) 189-199

2. Price, A., Kosaka, K. and Chatterjee, S.: A Key Pre-distribution Scheme for Wireless Sensor Networks. Wireless Telecommunications Symposium (2005) 253-260

3. Stajano, E., Anderson, R.: The Resurrecting Duckling: Security Issues in Ad-Hoc Wireless Networks. The Seventh International Workshop on Security Protocols (1999)

4. Ramkumar, M., Memon, N., Simha, R.: Pre-Loaded Key Based Multicast and Broadcast Authentication in Mobile Ad-Hoc Networks. Globecom, San Fransisco, CA, (2003)

5. Ramkumar, M., Memon, N.: On the Security of Random Key Predistribution Schemes. The Fifth Annual IEEE Information Assurance Workshop, NY (2004) 